\title{
Physical and electrical characterization of reduced graphene oxide synthesized adopting green route
}

\author{
TAPAS K GHOSH ${ }^{1,2}$, SHIRSHENDU GOPE ${ }^{1}$, DIPAK RANA ${ }^{3}$, INDRANIL ROY ${ }^{1}$, \\ GUNJAN SARKAR $^{1}$, SOURAV SADHUKHAN ${ }^{1}$, AMARTYA BHATTACHARYA ${ }^{1}$, \\ KRISHNENDU PRAMANIK ${ }^{1}$, SANATAN CHATTOPADHYAY ${ }^{4}$, MUKUT CHAKRABORTY ${ }^{2}$ \\ and DIPANKAR CHATTOPADHYAY ${ }^{1, *}$ \\ ${ }^{1}$ Department of Polymer Science and Technology, University of Calcutta, 92 A.P.C. Road, Kolkata 700 009, India \\ ${ }^{2}$ Department of Chemistry, West Bengal State University, Barasat, Kolkata 700 126, India \\ ${ }^{3}$ Department of Chemical and Biological Engineering, Industrial Membrane Research Institute, University of Ottawa, 161 \\ Louis Pasteur St., Ottawa, ON, Canada K1N 6N5 \\ ${ }^{4}$ Department of Electronic Science, University of Calcutta, 92 A.P.C. Road, Kolkata 700 009, India
}

MS received 27 July 2015; accepted 19 October 2015

\begin{abstract}
Graphene and its related materials are important areas of research in recent years owing to their unique properties. The wide-range industrial application of graphene-related compounds has led to the development of novel and simple methods for the synthesis of graphene. In this paper, an environmentally friendly green synthesis for the partial reduction of graphene oxide (GO) to reduced GO (RGO) in a cost-effective single-step mechanism was reported. The method completely avoids the use of toxic and environmentally harmful reducing agents commonly used in the chemical reduction of GO to obtain RGO. The reduction of GO was carried out using aqueous leaf extracts of Paederia foetide L. The prepared GO and green RGO were characterized by ultraviolet-visible, Raman and Fourier transform infra-red spectroscopic analysis which showed a clear indication of the partial removal of oxygen-containing groups from the GO and the formation of RGO. The morphology of the green RGO was characterized by transmission electron and scanning electron microscopy. Dynamic light scattering was used for zeta potential measurement and correlated with the morphology of the sheets. Electrical conductivity was also measured to check the extent of reduction of GO to RGO.
\end{abstract}

Keywords. Green reduction; Paederia foetida L. leaf; reduced graphene oxide; electrical conductivity.

\section{Introduction}

Graphene is amongst the materials of choice in recent years owing to its excellent electronic, optical, mechanical and catalytic properties [1-5]. It is a flat monolayer of $\mathrm{sp}^{2}$ bonded carbon atoms firmly packed into a two-dimensional (2-D) honeycomb lattice and has attracted a great deal of scientific interest since its discovery in 2004 [6]. High chemical stability and unique electronic and mechanical properties of graphene cling to a great promise for potential applications in nanoelectronics, hybrid materials, $\mathrm{Li}$ ion batteries and sensors [7-11]. Graphene has become an essential material for many electronic devices such as panels, $\mathrm{p}-\mathrm{n}$ junctions, thin film transistors $[12,13]$, memory devices and solar cells [14]. Graphene and graphene-based materials have also been applied in drug delivery [15], bio-sensing, [16] and catalysis [17]. Thus, synthesis of graphene sheets in a simplified manner is of immense interest to the scientific community. Out of the various methods for the preparation of graphene oxide

\footnotetext{
*Author for correspondence (dipankar.chattopadhyay@gmail.com)
}

(GO) to reduced GO (RGO), solution-based chemical reduction is considered as one of the most efficient methods for low-cost and large-scale production.

Presently, there are several different methods to prepare graphene such as chemical vapour deposition [18], micromechanical exfoliation [19], epitaxial growth [20], cutting carbon nanotubes [21], direct sonication [22] and electrochemical approaches [23]. Although RGO can be prepared by chemical and thermal reduction [24-27] the synthesized RGO tends to form irreversible aggregation due to strong van der Walls forces between the RGO sheets which hampers its processability. In the case of thermal reduction, the reaction was accompanied by the exclusion of epoxy and carboxyl groups from GO. It is usually observed that the dry GO powder decomposes near $210^{\circ} \mathrm{C}$ with a considerable mass loss caused by a loss of oxygen from GO [28]. Among these reported methods, the solution-based reduction of GO has some advantages in terms of cost effectiveness as well as large-scale productivity, although the tendency of $\mathrm{p}-\mathrm{p}$ stacking between the chemically reduced GO sheets may lead to irreversible agglomeration. The above mentioned problem can be effectively eliminated by 
surface modifications of GO using small organic molecules, biomolecules and polymers [29-31]. However, the use of surface modifiers is challenging, as they tend to reduce the electrical conductivity of graphene due to their intrinsic insulating effect, which in turn limits the practical applicability of the surface-modified graphene in the field of nanoelectronics. This approach is also not favourable from the environmental and economic point of view. Thus, a natural and rapid path for its conversion after the reduction of GO is preferred.

Keeping the above points in mind, it is very important to find environmentally friendly and effective reducing agents for the reduction of GO to RGO [32]. Biomolecules such as vitamin C [33,34], amino acid [35], sodium citrate [36] and glucose [37] have also been reported as reducing agents to produce RGO. Various phyto extracts such as carrot root [1], green tea [38] and bacteria [39] have been used to produce RGO. Although the degree of reduction by the green method are lower than the hydrazine-based method, green reduction of GO finds a wide spread of acceptability in biological and biomedical fields. However, some of the reported methods have some disadvantages like slow reaction rates $[33,40]$, poor stability [41], etc., which may decline the quality of the product.

Considering these points, we have developed a facile and green route to synthesize RGO using aqueous leaf extract of Paederia foetide L. (PFL) tree, which is non-toxic, rich in carotene and vitamin $\mathrm{C}$ and can act as both a reducing and stabilizing agent. Alcoholic extract of PFL leaf has antiinflammatory [42], anti-diarial [43] and anti-tussive [44] activity.

The leaves are rich in carotene and vitamin $\mathrm{C}$; and contain a high amount of protein consisting of arginine, histidine, lysine, tyrosine, tryptophan, phenylalanine, cystine, methionine and valine. The leaves also contain a volatile oil having an offensive odor due to the presence of methyl mercaptan [45]. Hentriacontane, hentriacontanol, methyl mercaptan, ceryl alcohol, palmitic acid, sitosterol, stigmasterol, campesterol, ursolic acid and iridoid glycosides paederoside, paederosidic acid, scandoside, asperuloside and deacetylasperuloside have also been isolated from leaves and stems of PFL [46]. It has been reported in literature that this extract was used to reduce $\mathrm{AgNO}_{3}$ and prepare silver nanoparticles [47]. Most significantly, in comparison with other strong reducing agents used in GO reduction, this is readily available and has low environmental impact.

GO and RGO obtained by green reduction using PFL leaf extract is characterized by X-ray diffraction (XRD), Fourier transform infrared (FTIR), Raman spectroscopic analysis, field emission scanning electron microscopy (FESEM), ultra-violet spectroscopy (UV), transmission electron microscopy (TEM), thermo-gravimetric analysis (TGA) and zeta potential measurement. The electrical conductivity of GO and RGO has been measured to check the extent of reduction and restoration of electronic conjugation in RGO.

\section{Experimental}

\subsection{Materials}

Concentrated sulphuric acid $\left(98 \% \mathrm{H}_{2} \mathrm{SO}_{4}\right.$, GR grade), potassium permanganate $\left(\mathrm{KMnO}_{4}\right.$ purified), hydrogen peroxide solution $\left(30 \% \mathrm{H}_{2} \mathrm{O}_{2}\right)$, sodium nitrite $\left(\mathrm{NaNO}_{2}\right.$, extra pure) and sodium carbonate $\left(\mathrm{Na}_{2} \mathrm{CO}_{3}\right)$ were received from Merck Specialties Pvt. Ltd., India. Pristine graphite powder $(92 \%$ pure) was received from Sigma-Aldrich (MW $12.01 \mathrm{~g} \mathrm{~mol}^{-1}$, $\left.\mathrm{mp} 3652-3697^{\circ} \mathrm{C}\right)$. The PFL plant was collected from the local area of Shrirampur, Hooghly, West Bengal, India.

\subsection{Preparation of GO by Hummer's method}

The synthesis of GO from graphite powder was carried out by following the Hummers and Offeman method $[48,49]$. At first, $23 \mathrm{ml}$ of $98 \%$ concentrated $\mathrm{H}_{2} \mathrm{SO}_{4}$ was cooled down to $0^{\circ} \mathrm{C}$ in an ice bath. Separately, $1 \mathrm{~g}$ of graphite powder and $0.5 \mathrm{~g}$ of $\mathrm{NaNO}_{2}$ were mixed together. This mixture was then added to the cooled $\left(0^{\circ} \mathrm{C}\right)$ concentrated $\mathrm{H}_{2} \mathrm{SO}_{4}$ and kept under constant stirring in an ice bath for $45 \mathrm{~min}$, so that the chemicals would have sufficient time to mix with each other. Then, $3 \mathrm{~g}$ of $\mathrm{KMnO}_{4}$ was added slowly and gradually to the solution. The colour of the solution immediately turned greenish black from black. The solution was removed from the ice bath and allowed to attain room temperature. After the attainment of room temperature, the solution was kept in an oilbath at $80-90^{\circ} \mathrm{C}$ with constant stirring until the colour of the solution changed to brown. MilliQ water $(140 \mathrm{ml})$ was subsequently added followed by the addition of $7 \mathrm{ml}$ of $30 \% \mathrm{H}_{2} \mathrm{O}_{2}$ solution to terminate the reaction and kept under stirring for another $15 \mathrm{~min}$. The solution finally changed its colour from dark brown to yellow and was left to settle overnight. The product was then filtered. At this time, the $\mathrm{pH}$ of the solution was very close to 2 which indicated its high acidic character. Next, the product was centrifuged followed by repeated washing to remove the excess acid. Finally, the product was vacuum dried at $50^{\circ} \mathrm{C}$ to obtain a $\mathrm{GO}$ powder (figure 1).

\subsection{Preparation of PFL leaf extract}

PFL leaf extract was prepared by dissolving $20 \mathrm{~g}$ of freshly chopped PFL leaf in $500 \mathrm{ml}$ Millipore water, boiled for 15 min, and then cooled to get the extract. Next, the mixture was filtered through a Whatman 42 filter paper and the filtrate was collected as the extract.

\subsection{Reduction of GO by PFL leaf extract}

The extract was added to the aqueous dispersion of GO with continuous stirring for approximately $12 \mathrm{~h}$ at about $50^{\circ} \mathrm{C}$. The colour of the mixture changed from brown to deep black. Finally, after cooling the mixture to room temperature, it was filtered using a Whatman 42 filter paper and washed repeatedly with distilled water. Subsequently, the RGO was dried and collected in powdered form. 


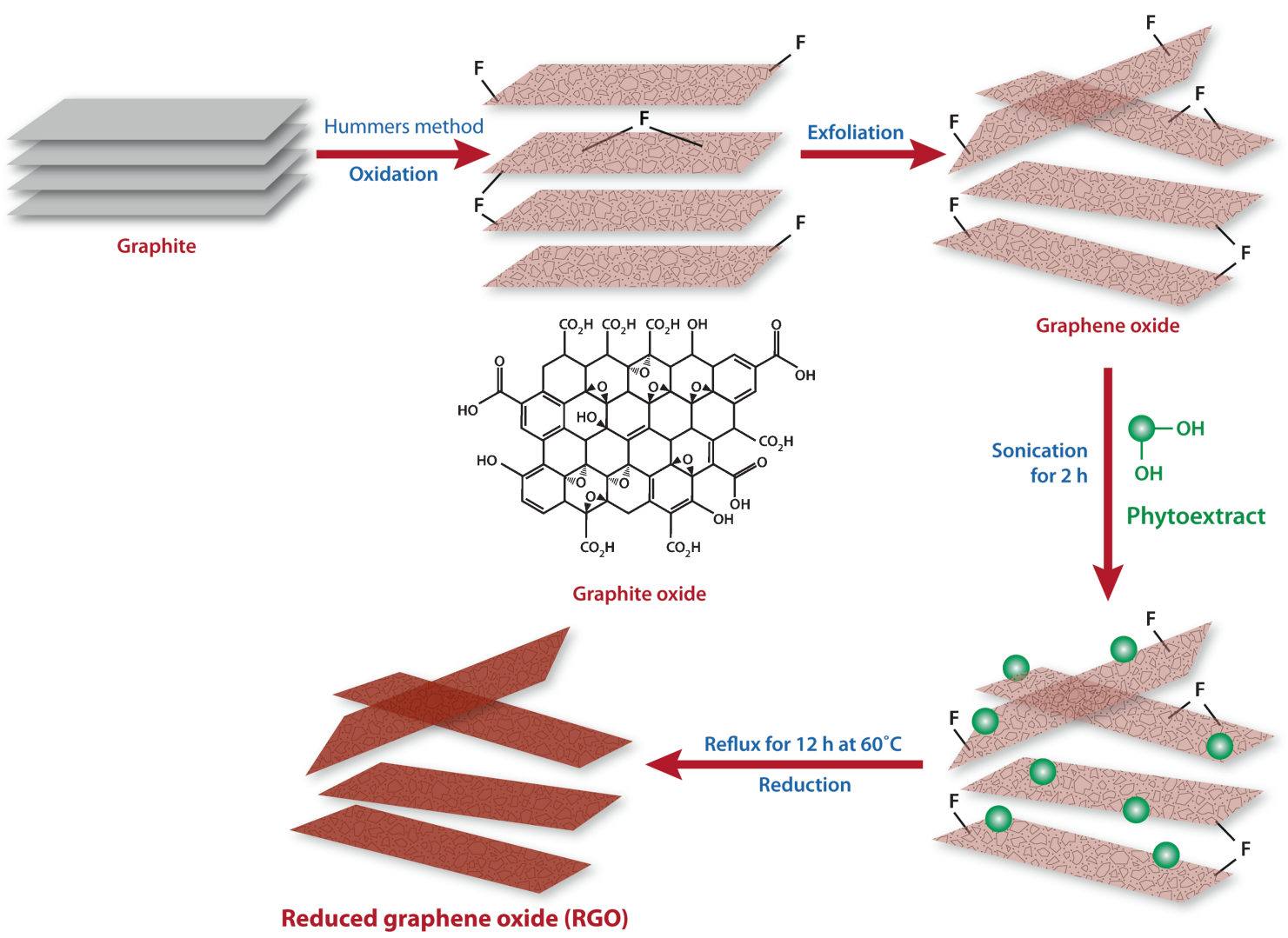

Figure 1. Schematic diagram of the total synthesis process.

\subsection{Characterization}

XRD analysis of GO and RGO were performed at room temperature by X-PERT-PRO Pan analytical diffractometer using $\mathrm{Cu} \mathrm{K} \alpha(\lambda=1.5406)$ as the $\mathrm{X}$-ray source at a generator voltage of $40 \mathrm{kV}$ and current of $30 \mathrm{~mA}$. The scanning rate was $1^{\circ} \mathrm{min}^{-1}$. From XRD data, the interlayer spacing of GO and RGO were calculated using Bragg's law as follows:

$$
d=\frac{\lambda}{2 \sin \theta}
$$

A UV-visible spectroscopy study was carried out using an Agilent 8453 Spectrophotometer, USA, in the wavelength range of $175-500 \mathrm{~nm}$.

FTIR spectroscopy was done to confirm the formation of GO and RGO and to identify the characteristic peaks of oxygen containing groups of GO such as epoxy, carbonyl, hydroxides, etc. and subsequent removal of these oxygenfunctional groups in the case of RGO after green reduction. FTIR spectrum was recorded using a PerkinElmer spectrum Express Version 1.03.00 instrument in the range of $250-4500 \mathrm{~cm}^{-1}$.

Raman spectroscopy is highly sensitive to the electronic structure and has proven to be an essential tool for the characterization of carbon-based materials, especially $\mathrm{C}=\mathrm{C}$ double bonds that lead to high Raman intensities. It is a useful tool to characterize GO and its reduced form is done by green reduction [49]. The Raman spectra was monitored using $1.96 \mathrm{eV}(633 \mathrm{~nm})$ line of a He-Ne laser in Horiba-JobinYvon Lab RAM HR 800 instrument by placing the sample solution into a semi-micro-stopper cuvette with an exposure time of $1 \mathrm{~s}$.

FESEM was used at different magnifications to observe the surface morphology of the GO and RGO samples. Before the analysis, the samples were gold coated to avoid electrical charging during examination. The FESEM study was performed in a Zeiss Auriga instrument.

A TEM (TEM-JEOL-JEM-2100 with a $200 \mathrm{kV}$ accelerating voltage) was used for the morphological analysis of the synthesized RGO. Samples for TEM analysis were prepared by drying a droplet of material suspension on a carbon-coated copper grid.

TGA analysis of HPMC and its nanocomposites films with GO were carried out in a Mettler-Toledo TGA/SDTA 851 thermal analyzer in a dynamic atmosphere of nitrogen (flow rate $=30 \mathrm{~cm}^{3} \mathrm{~min}^{-1}$ ). The samples were heated in an alumina crucible at a rate of $10^{\circ} \mathrm{C} \mathrm{min}{ }^{-1}$ over a temperature range of $50-800^{\circ} \mathrm{C}$.

The surface charge as well as stability of GO and RGO were examined by analysing the Zeta potential value. Zetapotential measurements were performed using a Zetasizer Nano-ZS90 System (Malvern Inc.).

Electrical conductivity of GO and RGO samples were measured using pellets having a thickness of $0.1 \mathrm{~cm}$ and diameter $0.5 \mathrm{~cm}$. The $I-V$ characteristics curves of the 
(a)

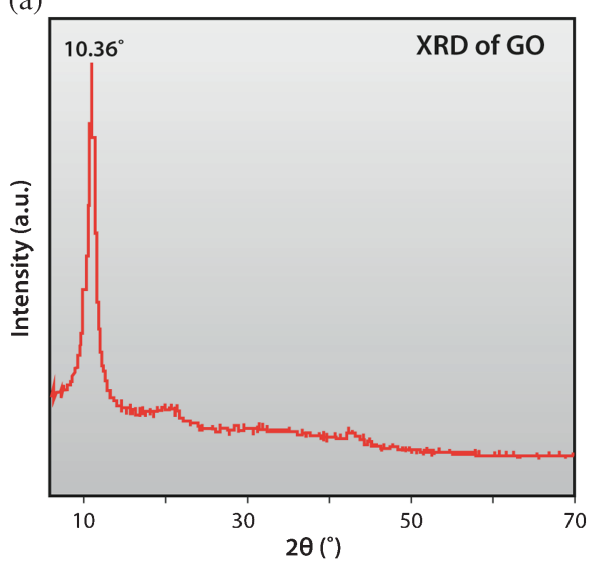

Figure 2. XRD patterns of GO and RGO.

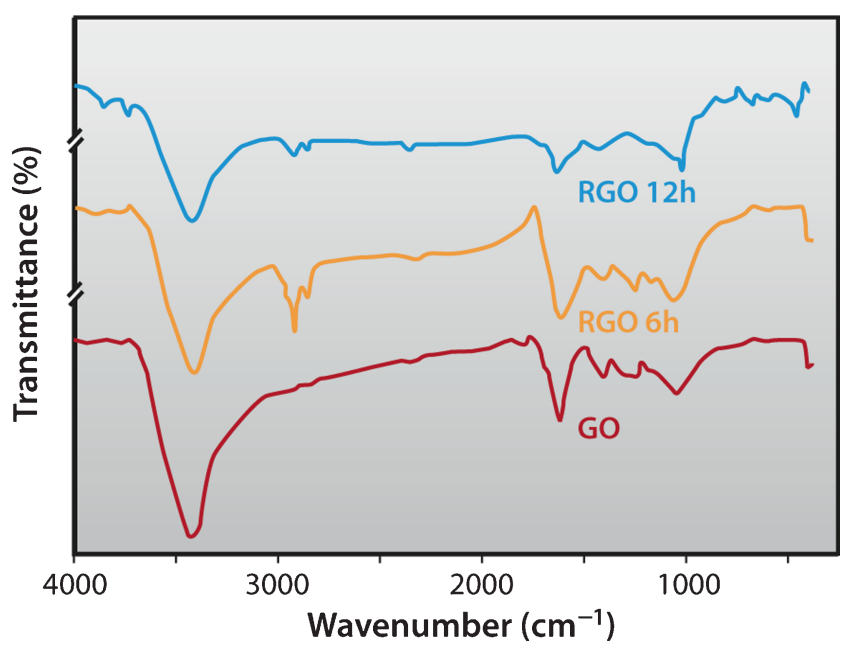

Figure 3. FTIR spectra of GO and RGO samples at different time interval.

samples of $0.01 \mathrm{~cm}^{2}$ were studied using two probe methods at room temperature by applying a voltage from -10 to $+10 \mathrm{~V}$ and the current was measured at each applied voltage.

\section{Results and discussion}

\subsection{XRD pattern}

XRD patterns of GO and RGO obtained by green reduction are shown in figure 2. From literature, we know that graphite shows a basal reflection peak at $2 \theta=26.6^{\circ}$ which corresponds to a $d$-spacing of $0.335 \mathrm{~nm}$ and after formation of GO by Hummer's method the peak shifts lower to $2 \theta=$ $10.36^{\circ}(d$-spacing corresponding to $0.8528 \mathrm{~nm})$, due to the intercalation of water molecules and incorporation of oxygen containing functional groups between the layer of graphite (figure 2a). The disappearance of the graphite peak at $26.6^{\circ}$ and subsequent appearance of the peak of GO at $10.36^{\circ}$ confirms the formation of GO by the Hummers method. After reduction of GO by PFL leaf extract, the obtained RGO

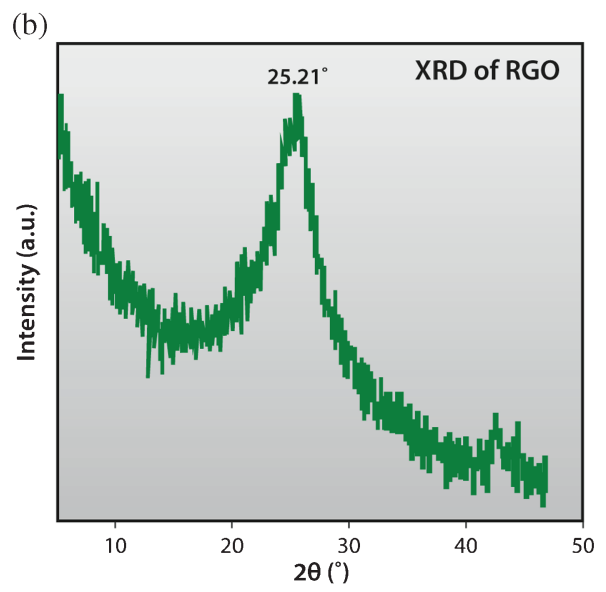

shows the reflection peak at $2 \theta=25.21^{\circ}$ corresponding to a $d$-spacing of $0.3528 \mathrm{~nm}$ (figure $2 \mathrm{~b}$ ). Peak is indexed to hexagonal C (JCPDS Card No. 75-1621) with its (002) peak at $25.5^{\circ}$. This is probably due to the restacking of the graphene layer. The complete disappearance of the characteristic peak of $\mathrm{GO}$ at $2 \theta=10.36^{\circ}$ in RGO indicates that the oxygen containing groups of GO have been removed efficiently to green reduction.

\subsection{FTIR spectra}

FTIR spectroscopy was performed to evaluate the deoxidization of GO by plant extract. Figure 3 shows the FTIR spectra of pure GO and RGO (after 6 and $12 \mathrm{~h}$ ) samples. The FTIR spectra of GO shows peaks corresponding to the oxygen functionalities present at 3432, 1710, 1628, 1394, 1261 and $1046 \mathrm{~cm}^{-1}$ due to $-\mathrm{OH}$ stretching, $-\mathrm{C}=\mathrm{O}$ stretching [50], $-\mathrm{C}=\mathrm{O},-\mathrm{O}-\mathrm{H}$ bending, $-\mathrm{C}-\mathrm{OH}$ stretching and $-\mathrm{C}-\mathrm{O}$ stretching vibration [51], respectively. The $\mathrm{C}=\mathrm{O}$ vibration peak at $1628 \mathrm{~cm}^{-1}$ and $\mathrm{O}-\mathrm{H}$ deformation peak at $1390 \mathrm{~cm}^{-1}$ of RGO decreases significantly from 6 to $12 \mathrm{~h}$ following the reduction of GO by the plant extract. The presence of two new peaks at 2860 and $2931 \mathrm{~cm}^{-1}$ are owing to the absorption of the plant extract containing phenolic compounds on the surface of graphene. So from the above observation it is clear that the increased reduction time leads to a greater removal of the oxygen functionalities from the GO surface, indicating a successful reduction of GO to RGO.

\subsection{UV spectroscopic analysis}

Figure 4 represents the UV-visible spectra of graphene oxide molecules dispersed in water. It is apparent from the spectra that pure GO shows two absorption peaks: one at $\sim 241$ $\mathrm{nm}$, due to the $\pi-\pi *$ transition of the $\mathrm{C}-\mathrm{C}$ bonds and another shoulder at $\sim 300 \mathrm{~nm}$ corresponds to the $\mathrm{n} \rightarrow \pi *$ transition of the $\mathrm{C}=\mathrm{O}$ bonds $[14,52]$. The absorption peak of the $\pi-\pi^{*}$ transition shifts to $262 \mathrm{~nm}$ in the case of RGO. This indicates that the groups on the GO surface are removed and a 


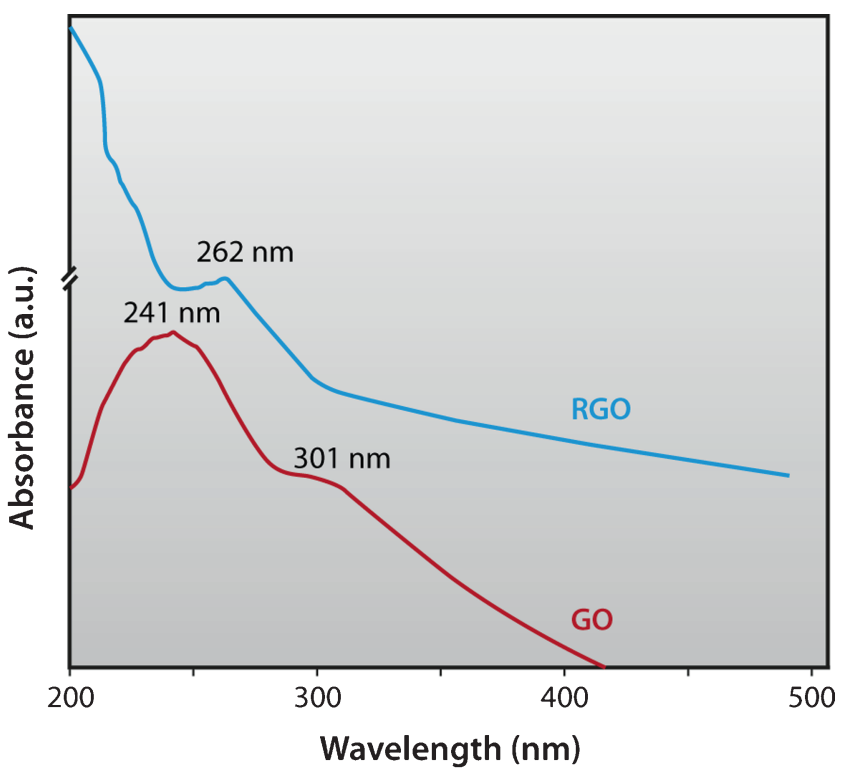

Figure 4. UV-spectra of GO suspension with concentration at $0.5 \mathrm{mg} \mathrm{ml}^{-1}$.

conjugated structure of RGO is formed, further confirming the reduction of GO to RGO by the green method. The red shift is probably due to the excitation of $\pi$ plasmon [53] of the resultant graphitic structure.

\subsection{Raman spectroscopy}

Raman spectroscopy is an essential tool [54] for the characterization of carbon-based materials, especially the $\mathrm{C}=\mathrm{C}$ double bonds that lead to high Raman intensities. Since Raman scattering is strongly dependent upon the electronic structure, it can be used to differentiate the difference between $\mathrm{GO}$ and its reduced form obtained after reduction using the PFL leaf extract. Raman spectrum of GO was observed to be significantly changed after the reduction from GO to RGO (figure 5). Raman spectra of GO and RGO are generally characterized by two main features. The $\mathrm{G}$ vibration mode, owing to the first-order scattering of $\mathrm{E}_{2} \mathrm{~g}$ phonons by $\mathrm{sp}^{2}$ carbon of GO and RGO were recorded at 1580 and $1575 \mathrm{~cm}^{-1}$, respectively, while the $\mathrm{D}$ vibration band was obtained from a breathing mode of $\kappa$-point photons [55] of $\mathrm{A}_{1} \mathrm{~g}$ symmetry of GO [51] and RGO appeared at 1334 and $1339 \mathrm{~cm}^{-1}$, respectively. After the reduction of $\mathrm{GO}$, the intensity ratio of the $\mathrm{D}$ band to the $\mathrm{G}$ band $\left(I_{\mathrm{D}} / I_{\mathrm{G}}\right)$ was increased significantly from 1.004 for GO to 1.037 for RGO. As the $\mathrm{D}$ band arises due to $\mathrm{sp}^{2}$ carbon cluster, the higher intensity of the D band suggests the presence of more isolated graphene domain in RGO in comparison to GO and therefore the removal of oxygen moieties from the former [14]. The presence of different types of functional groups in GO may prevent stacking of the graphene layers. However after reduction due to the removal of functional groups, the graphene layers can presumably be stacked together leading to the formation of a multilayer RGO.

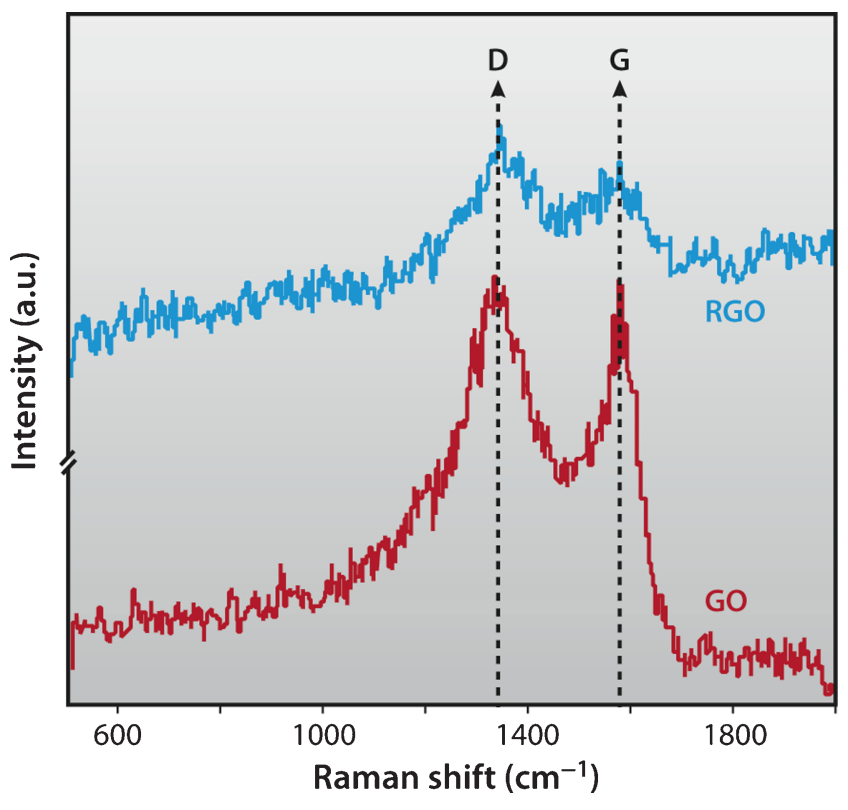

Figure 5. Raman analysis GO and RGO samples.

\subsection{DLS study}

To evaluate the stability of RGO in an aqueous solution, the measurement of Zeta potential was carried out. Zeta potential of GO and RGO has been carried out in a dynamic light scattering instrument to ascertain the stability of the solution (figure 6). GO shows an average zeta potential value of $-19.9 \pm 0.53 \mathrm{mV}$, indicating the existence of a sufficient amount of negative charge density arising from the carboxylic groups [56] present in GO. The reduction of GO with PFL leaf extract produces RGO with a negative charge density comparatively higher than that of GO after $6 \mathrm{~h}$ of reduction $(-21.7 \pm 0.47 \mathrm{mV})$ and after $12 \mathrm{~h}$ of reduction the zeta potential value is further increased to $-23.6 \pm 0.89 \mathrm{mV}$. The trend of zeta-potential data proved that the RGO possessed more surface negative charge density compared to GO. The electrostatic repulsion interaction of such negative charges was important and responsible for the stable dispersion of reduced graphene oxide sheets in an aqueous solution. The high charge density for both GO and RGO indicates that the dispersions obtained are quite stable in water. Furthermore, DLS measurement was performed to elucidate the state of the sheets in an aqueous solution. It was found that the average hydrodynamic diameter (AHD) of GO was $275 \mathrm{~nm}$ (figure 6). However, after the reduction of GO with PFL leaf extract after 6 and $12 \mathrm{~h}$, the AHD obtained were 178 and $154 \mathrm{~nm}$, respectively, under the same experimental conditions, which were relatively lower than that of GO. Since the particles were assumed to be spherical in the DLS analysis, the data in our case could not be taken to exactly evaluate the actual sizes of the 2-D structures, and could only be used to reflect the trend of changes in the interlayer distances of graphene $[57,58]$. Finally it may be said that after the reduction process, the size of RGO particles are approximately 1.5 times smaller than GO. The higher zeta potential values of RGO 

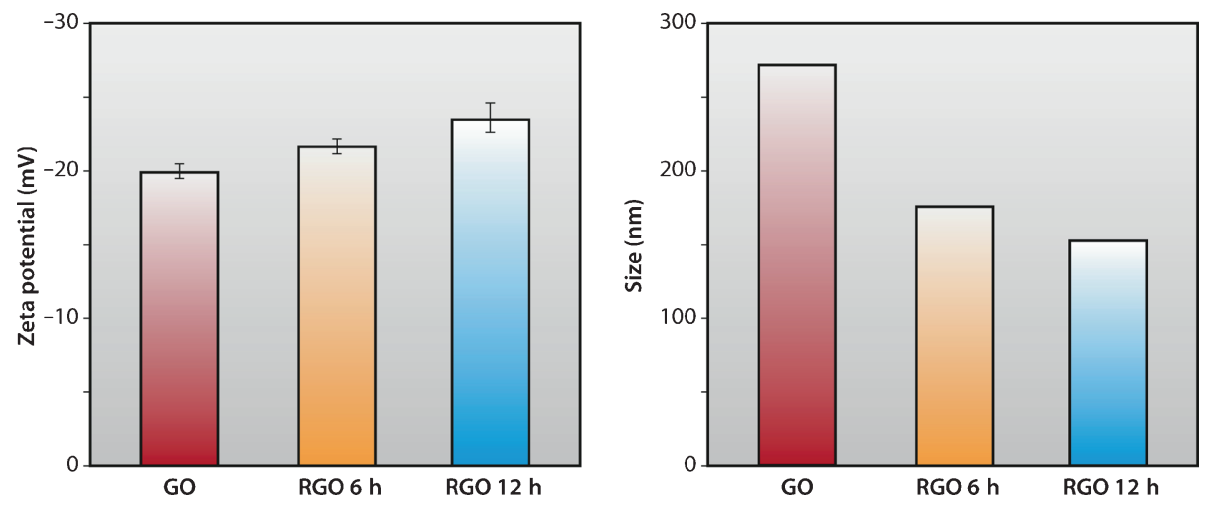

Figure 6. DLS study of GO and RGO at different time intervals.

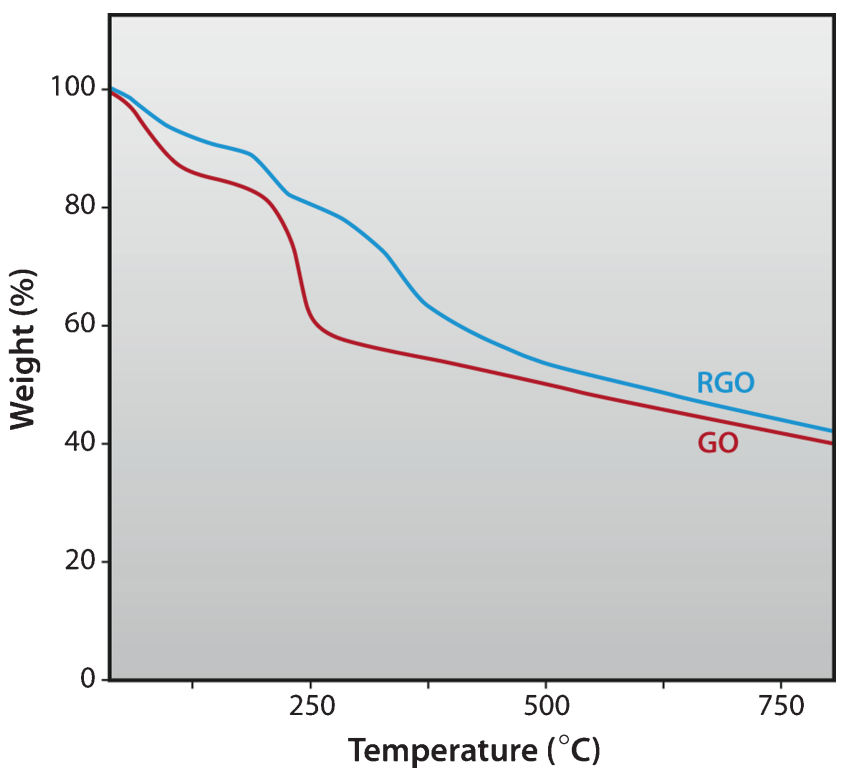

Figure 7. Thermo-gravimetric analysis of GO and RGO samples.

along with lower AHD values compared to GO, can probably be explained on the basis that reduction of GO leads to more closely stacked RGO layers. However, the chemicals present in the leaf extracts during reduction are adsorbed on the surface of RGO as they act as a stabilizing moiety also, leading to more stable dispersions and subsequently higher zeta potential values.

\subsection{Thermal study}

Thermal stability of GO and RGO were studied by TGA (figure 7). GO shows a little weight loss near $100^{\circ} \mathrm{C}$ but RGO showed no significant weight loss near $100^{\circ} \mathrm{C}$, as the samples were completely dried before testing to get rid of the influence of absorbed moisture on the test results. GO exhibits a double step degradation; the first step beginning at $184^{\circ} \mathrm{C}$ owing to the loss of hydroxyl and epoxy functional groups. In the second step, degradation is due to the pyrolysis of the remaining oxygen-containing groups as well as the burning of carbon present in the ring $[59,60]$. RGO shows only a
15-18 wt $\%$ loss up to $250^{\circ} \mathrm{C}$, which was much lower in comparison to that of the GO. The remarkable decrease in weight loss in the initial stages is due to the fact that RGO contains relatively much lower amount of oxygenated functional groups which accounts for the weight loss in the above mentioned temperature region. The approximate $20 \%$ less weight loss for RGO in the 1st step of degradation compared to GO is also an indication of the reduction process taking place during synthesis.

\subsection{FESEM and TEM analysis}

The morphology of GO and RGO were characterized using FESEM and TEM measurements. The dispersions were coated on aluminium foil, and SEM images were taken for each sample. The SEM images of the GO flakes shown in figure $8 \mathrm{a}$ shows the presence of individual sheets dispersed in the grid with low interaction between the sheets. However, the SEM images of the RGO material consists of individual sheets which are closely packed and aligned with each other as observed in earlier studies [40]. The RGO sheets have fairly large areas and appear to be more clumped and stacked with each other to form an interconnected network as shown in figure $8 \mathrm{~b}$. The TEM image (figure $8 \mathrm{c}$ ) of a portion of the RGO sheet under high magnification clearly shows an ultrathin sheet-like morphology.

\subsection{Electrical conductivity of $G O$ and $R G O$ samples}

The current-voltage characteristics of GO and RGO samples are plotted in figure 9. It is apparent from the plots that for a given voltage, GO samples exhibit a slightly lower current than RGO. It should be noted that the relatively lower current is indicative of the presence of functional groups in GO which have been partially removed after reduction using aqueous leaf extracts of PFL. Interestingly, both of the samples are considered in this current work, exhibit a significant hysteresis loop indicating their possibility to be used as memory elements. These results clearly indicates that GO is partially reduced by the aqueous leaf extracts of PFL and the electronic conjugation is restored after reduction. 

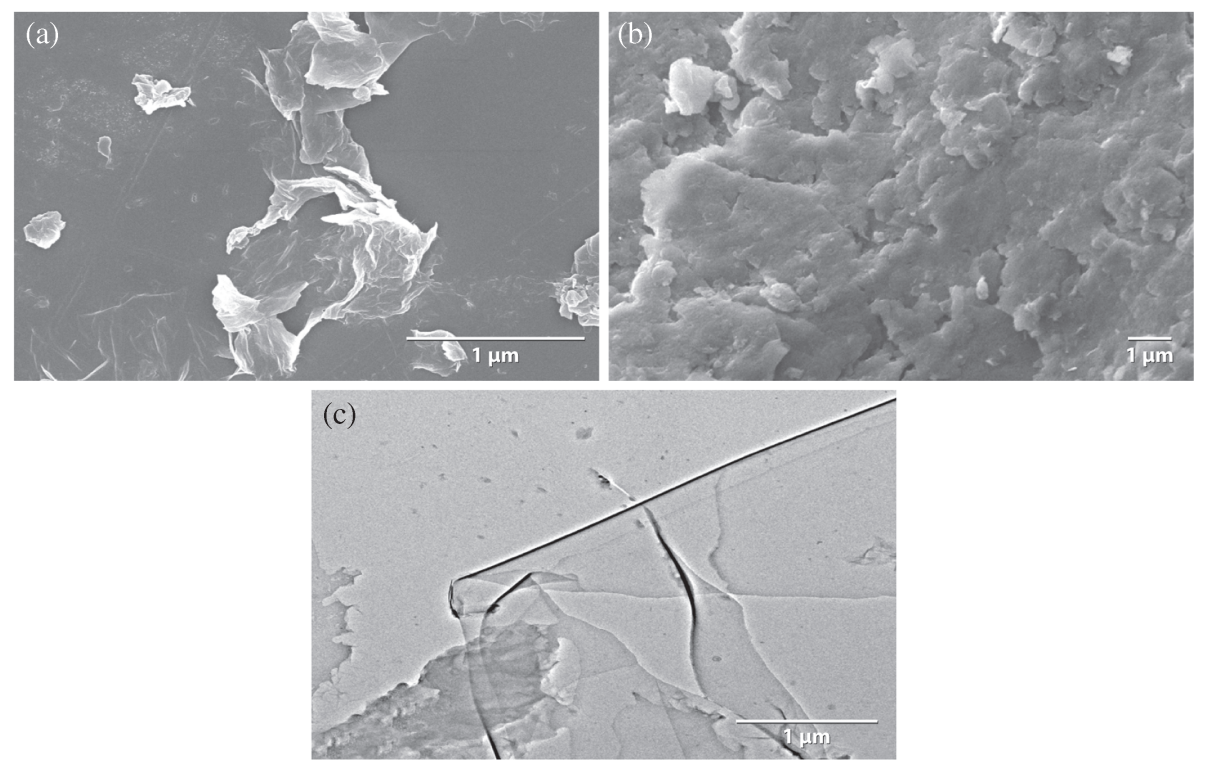

Figure 8. FESEM images of (a) GO, (b) RGO and (c) HRTEM image of RGO sheet.
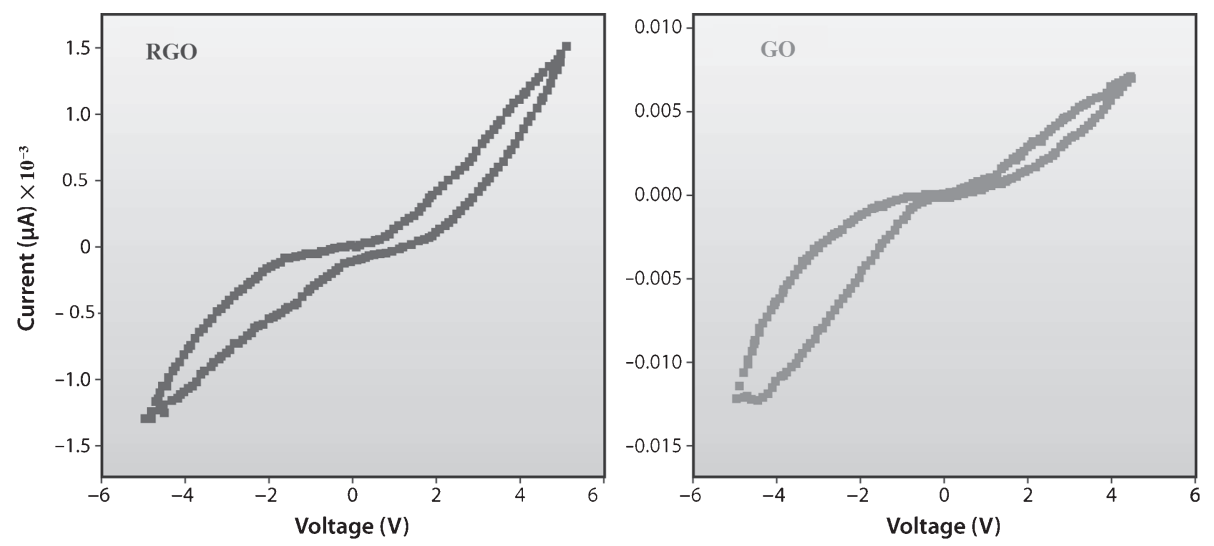

Figure 9. $I-V$ characteristics curve of $\mathrm{RGO}$ and $\mathrm{GO}$.

\section{Conclusion}

We have successfully demonstrated that the studied plant leaf extract (PFL leaf) has excellent potential to be used as a reducing agent for the reduction of GO to RGO with an environmental and eco-friendly benign synthetic protocol. The reduction was carried out in an aqueous medium at room temperature. The reduction of GO was confirmed by XRD, UV-vis, SEM and Raman spectroscopy. SEM and TEM images indicate that the RGO materials are layered and are formed into individual sheets, which are closely aligned with each other. FTIR analysis shows the deoxidization of oxygen functionalities from the surface of GO. A significant hysteresis indicates the possible use as memory devices. The main advantages of this technique over traditional chemical reaction are its cost effectiveness, environmentally friendly approach and easy product isolation procedure.

\section{Acknowledgements}

Roy likes to thank the TEQIP, University of Calcutta, for his fellowship. Sarkar likes to thank UGC, Government of India, in Rajiv Gandhi National Fellowship Scheme for his fellowship. We thank the Centre for Research in Nanoscience and Nanotechnology, University of Calcutta, for providing FESEM and TEM facilities. We also like to thank DST for FIST support.

\section{References}

[1] Kulia T, Bose S, Khanra P, Mishra A K, Kim N H and Lee J H 2012 Carbon 50914

[2] Geim A K 2009 Science 3241530

[3] Zhang Y B, Tan Y W, Stormer H L and Kim P 2005 Nature 438201 
[4] Wu J, Pisula W and Müllen K 2007 Chem. Rev. 107718

[5] Allen M J, Tung V C and Kaner R B 2010 Chem. Rev. 110132

[6] Novoselov K S, Geim A K, Morozov S V, Jiang D, Zhang Y, Dubonos S V, Grigorieva I V and Firsov A A 2004 Science 306666

[7] Liu S, Tian J Q, Wang L and Sun X P 2011 Carbon 493158

[8] Kim K S, Zhao Y, Jang H, Lee S Y, Kim J K, Kim K S, Ahn J-H, Kim P, Choi J-Y and Hong B H 2009 Nature 457 706

[9] Jung J H, Cheon D S, Liu F, Lee K B and Seo T S 2010 Angew. Chem. Int. Ed. 495708

[10] Schedin F, Geim A K, Morozov S V, Hill E W, Blake P, Katsnelson M I and Novoselov K S 2007 Nat. Mater. 6 652

[11] Chae S J, Günes F, Kim K K, Kim E S, Han G H, Kim S M, Shin H-J, Yoon S-M, Choi J-Y, Park M H, Yang C W, Pribat D and Lee Y H 2009 Adv. Mater. 212328

[12] Watcharotone S, Dikin D A, Stankovich S, Piner R, Jung I, Dommett G H B, Evmenenko G, Wu S-E, Chen S-F, Liu C-P, Nguyen S T and Ruoff R S 2007 Nano Lett. 71888

[13] Eda G, Fanchini G and Chhowalla M 2008 Nat. Nanotechnol. 3270

[14] Thakur S and Karak N 2012 Carbon 505331

[15] Sun X, Liu Z, Welsher K, Robinson J T, Goodwin A, Zaric S and Dai H 2008 Nano Res. 1203

[16] Lu C H, Yang H H, Zhu C L, Chen X and Chen G-N 2009 Angew. Chem. Int. Ed. 484785

[17] Song Y, Qu K, Zhao C, Ren J and Qu X 2010 Adv. Mater. 22 2206

[18] Wei D C, Liu Y Q, Wang Y, Zhang H L, Huang L P and Yu G 2009 Nano Lett. 91752

[19] Tang Z H, Zhuang J and Wang X 2010 Langmuir 269045

[20] Shivaraman S, Barton R A, Yu X, Alden J, Herman L H, Chandrashekhar M V S, Park J and McEuen P L 2009 Nano Lett. 93100

[21] Jiao L, Zhang L, Wang X, Diankov G and Dai H 2009 Nature 458877

[22] Lotya M, Hernandez Y, King P J, Smith R J, Nicolosi V, Karlsson L S, Blighe F M and De S 2009 J. Am. Chem. Soc. 1313611

[23] Wang Z, Zhou Z, Zhang J, Boey F and Zhang H 2009 J. Phys. Chem. C 11314071

[24] Park S and Ruoff R S 2009 Nat. Nanotechnol. 4217

[25] Stankovich S, Piner R D, Chen X, Wu N, Nguyen S T and Ruoff R S 2006 J. Mater. Chem. 16155

[26] Zhou Y, Bao Q, Tang L A L, Zhong Y and Loh K P 2009 Chem. Mater. 212950

[27] Wang H, Robinson J T, Li X and Dai H 2009 J. Am. Chem. Soc. 1319910

[28] Lerf A, He H, Forster M and Klinowski J 1998 J. Phys. Chem. B 1024477

[29] Si Y and Samulski T 2008 Nano Lett. 81679

[30] Shan C, Yang H, Han D, Zhang Q, Ivaska A and Liu N 2009 Langmuir 2512030

[31] Bai H, Xu Y, Zhao L, Li C and Shi G 2009 Chem. Commun. 451667

[32] Loh K P, Bao Q, Eda G and Chhowalla M 2010 Nat. Chem. 2 1015
[33] Zhang J, Yang H, Shen G, Cheng P, Zhang J and Guo S 2010 Chem. Commun. 461112

[34] Gao J, Liu F, Liu Y, Ma N, Wang Z and Zhang X 2010 Chem. Mater. 222213

[35] Shan C, Yang H, Han D, Zhang Q, Ivaska A and Niu L 2009 Langmuir 2512030

[36] Zhang Z, Chen H, Xing C, Guo M, Xu F, Wang X, Gruber H J, Zhang B and Tang J 2011 Nano Res. 4599

[37] Akhavan O, Ghaderi E, Aghayee S, Fereydooni Y and Talebi A 2012 J. Mater. Chem. 2213773

[38] Wang Y, Shi Z and Yin J 2011 ACS Appl. Mater. Interfaces 3 1127

[39] Gurunathan S, Han J W, Eppakayala V and Kim J-H 2013 Colloids Surf. B: Biointerfaces 102772

[40] Lee D Y, Khatun Z, Lee J-H, Lee Y and In I 2012 Biomacromolecules 12336

[41] Fernández-Merino M J, Guardia L, Paredes J I, Villar-Rodil S, Solis-Fernández P, Martinez-Alonso A and Tascón J M D 2010 J. Phys. Chem. C 1146426

[42] De S, Ravishankar B and Bhavsar G C 1994 J. Ethnopharmacol. 4331

[43] Afroz S, Alamgir M, Khan M T H, Jabbar S, Nahar N and Choudhuri M S K 2006 J. Ethnopharmacol. 105125

[44] Nosál'ová G, Mokrý J, Ather A and Khan M T H 2007 Acta Vet. Brno. 7627

[45] Ghani A 2012 Medicinal Plants of Bangladesh with Chemical Constituents and Uses 2nd ed (Dhaka: Asiatic Society of Bangladesh)

[46] Osman H, Rahim A A, Isa N M, Naemah M and Bakhir N M 2009 Molecules 14970

[47] Mollick M M R, Bhowmick B, Maity D, Mondal D, Bain M K, Bankura K, Sarkar J, Rana D, Acharya K and Chattopadhyay 2012 Int. J. Green Nanotechnol. 4230

[48] Hummers W S Jr and Offerman R E 1958 J. Am. Chem. Soc. 801339

[49] Fan W, Lai Q, Zhang Q and Wang Y 2011 J. Phys. Chem. C 11510694

[50] Nethravathi C and Rajamathi M 2008 Carbon 461994

[51] Goncalves G, Marques P A A P, Granadeiro C M, Nogueira H I S, Singh M K and Grásio J 2009 Chem. Mater. 21 4796

[52] Khanra P, Kuila T, Kim N, Bae S H, Yu D S and Lee J H 2012 Chem. Eng. J. 183526

[53] Carvalho A J F, Curvelo A A S and Agnelli J A M 2001 Carbohydr. Polym. 45189

[54] Gardiner D J, Graves P R (Eds) 1989 Practical Raman Spectroscopy (Berlin, Germany: Springer-Verlag)

[55] Lin Z, Yao Y, Li Z, Liu Y, Li Z and Wong C-P 2010 J. Phys. Chem. C 11414819

[56] Wang Y, Zhen S J, Zhang Y, Li Y F and Huang C Z 2011 J. Phys. Chem. C 11512815

[57] Wang Y, Li Y F, Wang J, Sang Y and Huang C Z 2010 Chem. Commun. 461332

[58] Wang Y, Chen L Q, Li Y F, Zhao X J, Peng L and Huang C Z 2010 Nanotechnology 21305601

[59] Jeong H K, Lee Y P, Jin M H, Kim E S, Bae J J and Lee Y H 2009 Chem. Phys. Lett. 470255

[60] Chen W, Yan L and Bangal P R 2010 Carbon 481146 